\title{
STABILITY AND CLOSED GRAPH THEOREMS IN CLASSES OF BORNOLOGICAL SPACES
}

\author{
by T. K. MUKHERJEE and W. H. SUMMERS
}

(Received 18 January, 1981; revised 27 August, 1981)

In the general theory of locally convex spaces, the idea of inductive limit is pervasive, with quotient spaces and the less obvious notion of direct sum being among the instances. Bornological spaces provide another important example. As is well known (cf. [7]), a Hausdorff locally convex space $E$ is bornological if, and only if, $E$ is an inductive limit of normed vector spaces. Going even further in this direction, a complete Hausdorff bornological space is an inductive limit of Banach spaces.

Inductive limits of Banach spaces form a distinguished subclass of the class of all bornological spaces; members of this subclass are termed ultrabornological (cf. [10]). Ultrabornological spaces, being inductive limits of barrelled spaces, are also barrelled. Moreover just as barrelled spaces can be characterized by a closed graph theorem [6], $\mathrm{M}$. De Wilde [2] has characterized ultrabornological spaces in terms of a closed graph theorem. In a barrelled space, every vector subspace of countable codimension is again barrelled [9], [11]; as M. Valdivia [17] has shown, however, unlike barrelled spaces, ultrabornological spaces can even contain hyperplanes which fail to be ultrabornological. At this point, it seems pertinent to ask whether it is possible to identify a larger collection of (barrelled and) bornological spaces which would exhibit improved stability properties, while retaining the nicer characteristics of ultrabornological spaces. Our present purpose is to show that this is indeed the case by introducing a class of barrelled and bornological spaces which we shall term hyperbornological. In so doing, we actually provide a general approach to describing and studying inductive limit generated subclasses of the bornological spaces, and then proceed to develop permanence and stability properties of these collections, as well as provide alternative characterizations including one in terms of a closed graph theorem.

1. Classes of bornological spaces. A subcollection $\mathscr{L}$ of the class $\mathcal{N}=\mathcal{N}(\mathbb{K})$ of all normed vector spaces (over the field $\mathbb{K} \in\{\mathbb{R}, \mathbb{C}\}$ ) will be called a bornology class (or $b$-class) whenever the following conditions are satisfied:

(i) $\mathscr{L}_{f} \subseteq \mathscr{L}$, where $\mathscr{L}_{\mathrm{f}}$ denotes the collection of all finite dimensional members of $\mathcal{N}$;

(ii) if $E \in \mathscr{L}$ and if $M$ is a closed vector subspace of $E$, then $E / M \in \mathscr{L}$;

(iii) if $\left\{E_{k}\right\}_{k=1}^{n}$ is any finite subset of $\mathscr{L}$, then $\prod_{k=1}^{n} E_{k} \in \mathscr{L}$.

Of course, $\mathcal{N}$ is the largest bornology class, while $\mathscr{L}_{f}$ is the smallest. Other examples include the collection $\mathscr{L}_{B}$ of all $E \in \mathcal{N}$ such that $E$ is a Banach space,

$$
\mathscr{L}_{r}=\left\{E \in \mathscr{L}_{\mathbf{B}}: E \text { is reflexive }\right\},
$$

and the collection $\mathscr{L}_{\mathrm{t}}$ consisting of all barrelled members of $\mathcal{N}$. We note in passing that

$$
\mathscr{L}_{f} \varsubsetneqq \mathscr{L}_{\mathrm{r}} \varsubsetneqq \mathscr{L}_{B} \varsubsetneqq \mathscr{L}_{\mathrm{t}} \varsubsetneqq \mathcal{N} \text {. }
$$

Glasgow Math. J. 23 (1982) 151-162. 
Let $E$ be a Hausdorff locally convex (topological vector) space. If $B$ is a (nonvoid) absolutely convex bounded subset of $E$, then $E(B)=\bigcup_{k=1}^{\infty} k B$ is a vector subspace of $E$ and the gauge $p_{B}$ of $B$ in $E(B)$ is a norm on $E(B)$; the normed space $\left(E(B), p_{B}\right)$ will hereafter be denoted by $E_{\mathrm{B}}$. Now, if $\mathscr{L}$ is a b-class, then a set $A \subseteq E$ will be termed $\mathscr{L}$-bounded whenever there exists an absolutely convex bounded set $B$ in $E$ such that $A \subseteq B$ and $E_{B}$ is topologically isomorphic to some $F \in \mathscr{L}$; the collection of all $\mathscr{L}$-bounded subsets of $E$ will be denoted by $\mathscr{L}(E)$.

Some properties of $\mathscr{L}$-bounded sets are listed below. Since verification is straightforward, it will be omitted.

Propostrion 1.1. Let $E$ be a Hausdorff locally convex space, and let $\mathscr{L}$ be a $b$-class.

(i) If $A \in \mathscr{L}(E)$, then $A$ is bounded, while every subset of $A$, the convex hull $\Gamma(A)$ of $A$, and the absolutely convex hull $\Gamma_{a}(A)$ of $A$ also belong to $\mathscr{L}(E)$;

(ii) if $A, B \in \mathscr{L}(E)$ and $\lambda \in \mathbb{K}$, then $\lambda A, A+B$, and $A \cup B$ are all in $\mathscr{L}(E)$;

(iii) $E=\cup\{A: A \in \mathscr{L}(E)\}$. [4].

In other words, $\mathscr{L}(E)$ is a convex vector bornology in the sense of H. Hogbe-Nlend

Given two b-classes $\mathscr{L}_{1}$ and $\mathscr{L}_{2}$, if each $E \in \mathscr{L}_{1}$ is topologically isomorphic to some $F \in \mathscr{L}_{2}$, then we will write $\mathscr{L}_{1} \leq \mathscr{L}_{2}$. Since $\mathscr{L}_{1}(E)$ will coincide with $\mathscr{L}_{2}(E)$ for every Hausdorff locally convex space $E$ if, and only if, $\mathscr{L}_{1} \leq \mathscr{L}_{2}$ and $\mathscr{L}_{2} \leq \mathscr{L}_{1}$, let us agree to identify $\mathscr{L}_{1}$ and $\mathscr{L}_{2}$ whenever both $\mathscr{L}_{1} \leq \mathscr{L}_{2}$ and $\mathscr{L}_{2} \leq \mathscr{L}_{1}$. With this understanding, we shall proceed to describe the classes of bornological spaces to which we alluded earlier.

Defintrion. A Hausdorff locally convex space $E$ will be termed $\mathscr{L}$-bornological, where $\mathscr{L}$ is a b-class, if each absolutely convex subset $U$ of $E$ which absorbs every $A \in \mathscr{L}(E)$ is a neighborhood (of zero).

If $\mathscr{L}_{1}$ and $\mathscr{L}_{2}$ are b-classes with $\mathscr{L}_{1} \leq \mathscr{L}_{2}$, then every $\mathscr{L}_{1}$-bornological space is clearly $\mathscr{L}_{2}$-bornological. Moreover, a Hausdorff locally convex space $E$ is $\mathcal{N}$-bornological if, and only if, $E$ is bornological, and therefore, for any b-class $\mathscr{L}$, every $\mathscr{L}$-bornological space is indeed bornological. It is also easy to check that every member of a given b-class $\mathscr{L}$ is $\mathscr{L}$-bornological.

THEOREM 1.2. Let $E$ be a Hausdorff locally convex space. Then, given a $b$-class $\mathscr{L}, E$ is $\mathscr{L}$-bornological if, and only if, $E$ is an inductive limit of normed vector spaces belonging to $\mathscr{L}$.

Proof. Assuming that $E$ is $\mathscr{L}$-bornological, let $\Lambda$ be the collection of all absolutely convex members $B$ of $\mathscr{L}(E)$ such that $E_{B}$ is topologically isomorphic to some $F_{B} \in \mathscr{L}$. Then, clearly,

$$
E=\underset{B \rightarrow}{\operatorname{ind}}\left\{E_{B}: B \in \Lambda\right\}=\underset{B \rightarrow}{\operatorname{ind}}\left\{F_{B}: B \in \Lambda\right\} .
$$

On the other hand, suppose that $E=\operatorname{ind}_{\lambda \rightarrow}\left\{E_{\lambda}: \lambda \in \Lambda\right\}$, where $E_{\lambda} \in \mathscr{L}, \lambda \in \Lambda$, and note that there is no loss of generality in further assuming that each $E_{\lambda} \subseteq E$. Now, if $U$ is an 
absolutely convex subset of $E$ which absorbs every $A \in \mathscr{L}(E)$, then $U$ necessarily absorbs the (closed) unit ball of $E_{\lambda}, \lambda \in \Lambda$, whereby $U$ is a neighborhood in $E$.

COROLlary 1.3. Let $E$ be a Hausdorff locally convex space. Then

(i) $E$ is ultrabornological if, and only if, $E$ is $\mathscr{L}_{\mathrm{B}}$-bornological;

(ii) $E$ is $\mathscr{L}_{f}$-bornological if, and only if, $E$ has its finest locally convex topology.

Comment on $\mathscr{L}_{r}$-bornological spaces will be reserved until Section 5 . In the meantime, the focus will be on the $\mathscr{L}_{t}$-bornological spaces; members of this latter class will also be called hyperbornological spaces. Since $\mathscr{L}_{B} \leq \mathscr{L}_{t}$, every ultrabornological space is hyperbornological. However, as we shall later demonstrate, the converse is not true.

COROLLARY 1.4. Every hyperbornological space is barrelled and bornological.

If $\mathscr{L}$ is a b-class such that every $\mathscr{L}$-bornological space is barrelled, then each $E \in \mathscr{L}$ is barrelled whence $\mathscr{L} \leq \mathscr{L}_{1}$; the converse assertion is an immediate consequence of Theorem 1.2. Unfortunately, though, the hyperbornological spaces do not exhaust the class of barrelled and bornological spaces (cf. [15]). Indeed, as we will see in Example 5.5, even more can be said in this direction.

Fixing a b-class $\mathscr{L}$, let $E$ be a Hausdorff locally convex space. We will say that $E$ is $\mathscr{L}$-quasibarrelled if every barrel in $E$ which absorbs every $A \in \mathscr{L}(E)$ is a neighborhood. Further; a subset $\mathscr{B}$ of $\mathscr{L}(E)$ will be termed a generating set (for the $\mathscr{L}$-bounded subsets of $E$ ) if (a) each $B \in \mathscr{B}$ is absolutely convex and (b) every absolutely convex set in $E$ which absorbs each $B \in \mathscr{B}$ also absorbs every $A \in \mathscr{L}(E)$; if, in addition, each $B \in \mathscr{B}$ is closed in $E$, we will refer to $\mathscr{B}$ as a closed generating set. For example, the collection of all absolutely convex compact subsets of $E$ is readily seen to be a closed generating set for $\mathscr{L}_{B}(E)$.

PROPOSITION 1.5. The following are equivalent for a Hausdorff locally convex space E:

(i) $E$ is barrelled;

(ii) $E$ is $\mathscr{L}_{\mathrm{B}}$-quasibarrelled;

(iii) $E$ is $\mathscr{L}$-quasibarrelled with respect to a b-class $\mathscr{L}$ such that there is a generating set $\mathscr{B}$ for $\mathscr{L}(E)$, where each $B \in \mathscr{B}$ is sequentially complete;

(iv) $E$ is $\mathscr{L}$-quasibarrelled for some b-class $\mathscr{L}$ with $\mathscr{L} \leq \mathscr{L}_{\text {. }}$.

Proof. That (i) implies (ii) is obvious, while the implication (ii) $\Rightarrow$ (iii) follows from the remark above. Assume that (iii) holds, and let $U$ be a barrel in $E$ which absorbs every $A \in \mathscr{L}_{\mathrm{t}}(E)$. Since $E_{\mathrm{B}}$ is a Banach space for each $B \in \mathscr{B}, \mathscr{B} \subseteq \mathscr{L}_{\mathrm{t}}(E)$ wherefore $U$ absorbs every $A \in \mathscr{L}(E)$, i.e. $U$ is a neighborhood. To see that (iv) $\Rightarrow$ (i), let $U$ be a barrel in $E$, take $A \in \mathscr{L}(E)$, and choose an absolutely convex set $B \in \mathscr{L}(E)$ so that $A \subseteq B$ and $E_{B}$ is barrelled. Since the canonical embedding $\psi_{B}: E_{B} \rightarrow E$ is continuous, $\psi_{B}^{-1}(U)$ is a barrel in $E_{\mathrm{B}}$. But this implies $U$ absorbs $B$, and the proof is complete.

For any b-class $\mathscr{L}$, of course, every $\mathscr{L}$-bornological space is $\mathscr{L}$-quasibarrelled, while each $\mathscr{L}$-quasibarrelled space is quasibarrelled. In Section 5 , we will consider the question of whether being $\mathscr{L}$-quasibarrelled does indeed offer a middle ground between the notions of barrelled and quasibarrelled. 
2. Local convergence. The concept of local (or Mackey) convergence has proved useful in studying continuity of linear mappings on bornological spaces (cf. [5]), and so it is not surprising that the same is true for the obvious modification in the context of this article. To be precise, for a given b-class $\mathscr{L}$, we shall say that a sequence $\left\{x_{n}\right\}_{n=1}^{\infty}$ in a Hausdorff locally convex space $E$ is $\mathscr{L}$-locally convergent to $x \in E$ if there exists an absolutely convex set $B \in \mathscr{L}(E)$ such that $x_{n}, n=1,2, \ldots$, and $x$ all belong to $E_{B}$ and $\left\{x_{n}\right\}_{n=1}^{\infty}$ converges to $x$ in (the normed vector space) $E_{B}$. A sequence $\left\{x_{n}\right\}_{n=1}^{\infty}$ in $E$ which $\mathscr{L}$-locally converges to zero will be termed $\mathscr{L}$-locally null. Further, a linear mapping $f$ from $E$ into a locally convex space $F$ will be called $\mathscr{L}$-locally bounded if $f(B)$ is bounded for each $B \in \mathscr{L}(E)$.

Since it is straightforward to check that an absolutely convex set $U$ in $E$ absorbs every $B \in \mathscr{L}(E)$ exactly when $U$ absorbs each $\mathscr{L}$-locally null sequence, and since the collection of all absolutely convex subsets of $E$ which absorb every $B \in \mathscr{L}(E)$ is a base (at zero) for a locally convex topology $\tau(\mathscr{L})$ on $E$ which is at least as fine as the initial topology on $E$, we have the following natural analogue of a standard result for bornological spaces.

Proposition 2.1. The following are equivalent for $a b$-class $\mathscr{L}$ and Hausdorff locally convex space $E$ :

(i) $E$ is $\mathscr{L}$-bornological;

(ii) every $\mathscr{L}$-locally bounded linear map f from $E$ into any locally convex space $F$ is continuous;

(iii) every absolutely convex subset $U$ of $E$ which absorbs each $\mathscr{L}$-locally null sequence is a neighborhood.

COROLlary 2.2. Assume that $\mathscr{L}$ is a $b$-class, and take $E$ to be $\mathscr{L}$-bornological. Then a linear mapping $f$ from $E$ into a locally convex space $F$ is continuous if, and only if, the image under $f$ of every $\mathscr{L}$-locally null sequence is bounded.

Corollary 2.3. For a given $b$-class $\mathscr{L}$, a Hausdorf locally convex space $E$ is $\mathscr{L}$-bornological if, and only if, every $\mathscr{L}$-locally bounded linear functional on $E$ is continuous and $E$ has the Mackey topology $\tau\left(E, E^{\prime}\right)$.

Proof. Since $\tau\left(E, E^{\prime}\right)$ is always coarser than $\tau(\mathscr{L})$, it suffices to note that, as a consequence of the Mackey-Arens theorem, $\tau\left(E, E^{\prime}\right)$ is finer than $\tau(\mathscr{L})$ whenever each $\mathscr{L}$-locally bounded linear functional on $E$ belongs to $E^{\prime}$.

The dual characterization of bornological spaces due to G. Köthe (cf. [5, p. 386]) also extends, although the statement is less than satisfactory.

TheOREM 2.4. Fixing a $b$-class $\mathscr{L}$, let $E$ be a Hausdorff locally convex space. Then $E$ is $\mathscr{L}$-bornological if, and only if, the following conditions are satisfied:

(i) E has its Mackey topology $\tau\left(E, E^{\prime}\right)$;

(ii) every absolutely convex subset of $E$ which absorbs each $B \in \mathscr{L}(E)$ likewise absorbs the closure of every $B \in \mathscr{L}(E)$; 
(iii) $E^{\prime}$ is complete in the topology $\sigma(\mathscr{L})$ of uniform convergence on all $\mathscr{L}$-locally null sequences.

Proof. If $E$ is $\mathscr{L}$-bornological, then Corollary 2.3 gives us that (i) holds, while (ii) follows since the closure of each $B \in \mathscr{L}(E)$ is bounded. For (iii), first note that a Cauchy net $\left\{f_{\lambda}\right\}$ in $\left(E^{\prime}, \sigma(\mathscr{L})\right)$ is a Cauchy net in the algebraic dual $E^{*}$ with its weak topology $\sigma\left(E^{*}, E\right)$ whereby $\left\{f_{\lambda}\right\}$ is $\sigma\left(E^{*}, E\right)$-convergent to some $f \in E^{*}$. A standard argument then shows that $f$ is $\mathscr{L}$-locally bounded, and hence $f \in E^{\prime}$ by Corollary 2.3 , and that $\left\{f_{\lambda}\right\}$ is $\sigma(\mathscr{L})$-convergent to $f$.

For the converse, again using Corollary 2.3 , it will suffice to show that each $\mathscr{L}$-locally bounded $f \in E^{*}$ belongs to $E^{\prime}$. Because of (ii), however, if $B \in \mathscr{L}(E)$ is absolutely convex and $f \in E^{*}$ is $\mathscr{L}$-locally bounded, then $f \mid E_{\bar{B}}$ is bounded. From this point, the argument exactly parallels Köthe's proof for the bornological case, and so we omit the details.

Given a b-class $\mathscr{L}$ and a Hausdorff locally convex space $E$, let $\mathscr{B}$ be any generating set for $\mathscr{L}(E)$. Then, since $f \in E^{*}$ is $\mathscr{L}$-locally bounded if, and only if, $f(B)$ is bounded for every $B \in \mathscr{B}$, Corollary 2.3 can be rephrased in terms of " $\mathscr{B}$-local boundedness." With this in mind, the proof of 2.4 can be obviously modified to yield the following variation. For convenience, we shall refer to a sequence $\left\{x_{n}\right\}$ in $E$ as being $\mathscr{B}(\mathscr{L})$-locally null whenever there exists some $B \in \mathscr{B}$ such that $\left\{x_{n}\right\}$ is a null sequence in $E_{B}$.

THeOREM 2.5. Fixing a $b$-class $\mathscr{L}$, let $E$ be a Hausdorff locally convex space such that $\mathscr{L}(E)$ has a closed generating set $\mathscr{B}$. Then $E$ is $\mathscr{L}$-bornological if, and only if, $E$ has its Mackey topology $\tau\left(E, E^{\prime}\right)$ and $E^{\prime}$ is complete in the topology $\sigma(\mathscr{B}(\mathscr{L})$ ) of uniform convergence on all $\mathscr{B}(\mathscr{L})$-locally null sequences.

CoRollary 2.6. A Hausdorff locally convex space $E$ is ultrabornological if, and only if, $E$ has the topology $\tau\left(E, E^{\prime}\right)$ and $E^{\prime}$ is $\sigma\left(\mathscr{B}\left(\mathscr{L}_{\mathscr{B}}\right)\right)$-complete, where $\mathscr{B}$ is the collection of all absolutely convex compact subsets of $E$.

In the setting of 2.6 , the $\mathscr{B}\left(\mathscr{L}_{B}\right)$-locally null sequences are just the sequences in $E$ which are fast convergent to zero in the sense of De Wilde (cf. [2]).

3. Stability properties. Our first observation is an immediate consequence of Theorem 1.2.

Proposition 3.1. For a b-class $\mathscr{L}$, every Hausdorff locally convex space which is an inductive limit of $\mathscr{L}$-bornological spaces is itself $\mathscr{L}$-bornological. In particular, any direct sum of $\mathscr{L}$-bornological spaces or any quotient of a $\mathscr{L}$-bornological space (by a closed vector subspace) is $\mathscr{L}$-bornological.

The situation for products is a bit more delicate. In view of Corollary 1.3, for instance, a denumerable product of $\mathscr{L}_{f}$-bornological spaces need not be $\mathscr{L}_{f}$-bornological, whereas countable products of bornological spaces are always bornological (cf. [5, p. $384]$ ).

For an infinite collection $\left\{E_{\lambda}: \lambda \in \Lambda\right\}$ of locally convex spaces, let $e$ denote the cardinality of $\Lambda$, and take $d$ to be an infinite cardinal with $d \leq e$. Then the subset of the 
topological product $E=\prod_{\lambda} E_{\lambda}$ consisting of those $x \in E$ such that $|\{\lambda \in \Lambda: x(\lambda) \neq 0\}| \leq d$ is a vector subspace of $E$ which, when equipped with the relative product topology, we shall denote by $E_{d}$; if $d=\aleph_{0}$, then $E_{d}$ is the $\Sigma$-product (with base point $0 \in E$ ) introduced by $H$. $H$. Corson [1]. Further, the topological product of $d$ copies of the scalar field $K$ will be denoted by $\omega_{d}$.

If, for each $\lambda \in \Lambda, E_{\lambda}$ is a Hausdorff locally convex space equipped with its Mackey topology $\tau\left(E_{\lambda}, E_{\lambda}^{\prime}\right)$, then the topological product $E=\prod_{\lambda} E_{\lambda}$ is known to have the topology $\tau\left(E, E^{\prime}\right)$. As we next note, this result can be extended to include our generalized $\Sigma$-products.

LEMMA 3.2. Let $\left\{E_{\lambda}: \lambda \in \Lambda\right\}$ be a family of Hausdorff locally convex spaces where, for each $\lambda \in \Lambda, E_{\lambda}$ has the topology $\tau\left(E_{\lambda}, E_{\lambda}^{\prime}\right)$. If $d$ is an infinite cardinal such that $d \leq|\Lambda|$, then $E_{d}$ has its Mackey topology $\tau\left(E_{d}, E_{d}^{\prime}\right)$.

Proof. Take $A$ in $E_{d}^{\prime}=E^{\prime}$ to be absolutely convex and $\sigma\left(E^{\prime}, E_{d}\right)$-compact. Since $A$ is $\sigma\left(E^{\prime}, E_{d}\right)$-bounded, and since any $x \in E$ which vanishes except on a countable subset of $\Lambda$ belongs to $E_{d}$, the projections of $A$ in $E_{\lambda}^{\prime}, \lambda \in \Lambda$, can be nontrivial in only finitely many instances whence $A$ is also $\sigma\left(E^{\prime}, E\right)$-compact.

TheOREM 3.3. Given a b-class $\mathscr{L}$ with $\mathscr{L}_{\mathbf{B}} \leq \mathscr{L}$, let $\left\{E_{\lambda}: \lambda \in \Lambda\right\}$ be a family of $\mathscr{L}$-bornological spaces (with $E_{\lambda} \neq\{0\}$ for every $\lambda \in \Lambda$ ), and assume that $d$ is an infinite cardinal such that $d \leq|\Lambda|$. Then $E_{d}$ is $\mathscr{L}$-bornological if, and only if, $\omega_{d}$ is bornological.

Proof. Assuming that $E_{d}$ is $\mathscr{L}$-bornological, choose $\Delta \subseteq \Lambda$ so that $|\Delta|=d$. Then $F=\prod_{\lambda \in \Delta} E_{\lambda}$ is topologically isomorphic to a quotient of $E_{d}$, and hence, from Proposition 3.1, we have that $F$ is bornological whereby so also is $\omega_{d}[5, \mathrm{p} .390]$. For the converse, let us begin by assuming that $f \in E_{d}^{*}$ is $\mathscr{L}$-locally bounded. Now, identifying each $E_{\lambda}$ with its image in $E_{d}$ under the natural injection mapping, put $A=\left\{\lambda \in \Lambda: f \mid E_{\lambda} \neq 0\right\}$. If $A$ were infinite, then there would exist a null sequence $\left\{x_{n}\right\}$ in $E_{d}$ such that $f\left(x_{n}\right)=n, n=1,2, \ldots$, and $B=\Gamma_{a}\left(\left\{x_{n}\right\}\right)$ is contained in a complete vector subspace of $E_{d}$. Since $\bar{B}$ would then be compact and therefore $\mathscr{L}$-bounded, however, this would contradict our choice of $f$. Thus, because $A$ is finite, $F=\prod_{\lambda \in A} E_{\lambda}$ is $\mathscr{L}$-bornological by Proposition 3.1 , while $f \mid F$ is $\mathscr{L}$-locally bounded and hence continuous by Corollary 2.3 . Consequently, there exists $g \in E_{d}^{\prime}$ so that $g|F=f| F$ and $g(x)=0$ whenever $x \in E_{d}$ with $x(\lambda)=0$ for all $\lambda \in A$; put $h=f-g$. Fixing $x \in E_{d}$, let $G_{\lambda}$ be the linear span of $x(\lambda), \lambda \in \Lambda$. Then $G=\prod_{\lambda} G_{\lambda}$ is a vector subspace of $E_{d}$ which, under the relative topology induced by $E_{d}$, is topologically isomorphic to a quotient of the bornological space $\omega_{d}$. Being complete, however, $\omega_{d}$ is ultrabornological and therefore $\mathscr{L}$-bornological whence $G$ is also $\mathscr{L}$-bornological. But this implies $h \mid G$ is continuous, and so, since $h(y)=0$ whenever $y$ belongs to the direct sum $\sum_{\lambda} \oplus E_{\lambda}$, we have $h \mid G=0$ whereby $h(x)=0$. Since $E_{d}$ has the topology $\tau\left(E_{d}, E_{d}^{\prime}\right)$ by Lemma 3.2, another application of Corollary 2.3 now serves to conclude the proof. 
Corollary 3.4. Given a b-class $\mathscr{L}$ with $\mathscr{L}_{\mathrm{B}} \leq \mathscr{L}$, let $\left\{E_{\lambda}: \lambda \in \Lambda\right\}$ be a collection of $\mathscr{L}$-bornological spaces. Then the topological product $E=\prod_{\lambda} E_{\lambda}$ is $\mathscr{L}$-bornological if, and only if, $E$ is bornological.

Proof. Necessity is obvious, as is sufficiency when $d=\left|\left\{\lambda \in \Lambda: E_{\lambda} \neq\{0\}\right\}\right|$ is finite. Assume, therefore, that $d$ is infinite and $E$ is bornological. Then $\omega_{d}$ is bornological [5, p. 390], and thus Theorem 3.3 yields that $E_{d}$, and hence $E$, is $\mathscr{L}$-bornological.

COROLlary 3.5. If $\left\{E_{\lambda}: \lambda \in \Lambda\right\}$ is a collection of hyperbornological (respectively, ultrabornological) spaces, then $E=\prod_{\lambda} E_{\lambda}$ is hyperbornological (respectively, ultrabornological) if, and only if, $E$ is bornological.

'Our next result is an immediate consequence of Theorem 3.3 and the Mackey-Ulam theorem.

THeOREM 3.6. Given a $b$-class $\mathscr{L}$ with $\mathscr{L}_{B} \leq \mathscr{L}$, let $\left\{E_{\lambda}: \lambda \in \Lambda\right\}$ be an infinite family of $\mathscr{L}$-bornological spaces. If $d$ is an infinite cardinal which is smaller than the first strongly inaccessible cardinal and such that $d \leq|\Lambda|$, then $E_{d}$ is $\mathscr{L}$-bornological. In particular, the $\Sigma$-product $E_{\aleph_{0}}$ is $\mathscr{L}$-bornological.

In [14], Valdivia establishes the fact that a $\Sigma$-product of barrelled and bornological spaces is (barrelled and) bornological; the preceding theorem asserts that, among other things, a $\Sigma$-product of bornological spaces is always bornological. The next observation also follows directly.

COROLlary 3.7. If $\left\{E_{\lambda}: \lambda \in \Lambda\right\}$ is an infinite collection of hyperbornological (respectively, ultrabornological) spaces, then the $\Sigma$-product $E_{\aleph_{0}}$ is hyperbornological (respectively, ultrabornological).

If a Hausdorff locally convex space $E$ contains a dense vector subspace $F$ which is barrelled in the induced topology, then $E$, of course, must itself be barrelled. However, as the work in [14] serves to demonstrate, if $F$ is bornological, or even ultrabornological, it need not follow that $E$ is bornological, but Valdivia has noted in [12] that a stronger notion of density does yield a positive result. Given a b-class $\mathscr{L}$, let us say that $F$ is $\mathscr{L}$-dense in $E$ whenever, for each $x \in E$, there exists a sequence $\left\{x_{n}\right\}$ in $F$ which is $\mathscr{L}$-locally convergent to $x$. Then, in our context, Valdivia's observation takes the following form.

Proposition 3.8. Let $E$ be a Hausdorff locally convex space and fix a $b$-class $\mathscr{L}$. If $E$ contains a $\mathscr{L}$-dense vector subspace $F$ which is $\mathscr{L}$-bornological in the topology induced by $E$, then $E$ is $\mathscr{L}$-bornological.

Proof. Utilize Corollary 2.3. It will clearly be enough to take $f \in E^{*}$ such that $f$ is $\mathscr{L}$-locally bounded and establish that $f=0$ under the additional assumption that $f \mid F=0$. To do this, fix $x \in E$, and choose a sequence $\left\{x_{n}\right\}$ in $F$ and an absolutely convex set $B \in \mathscr{L}(E)$ so that $\left\{x_{n}\right\}$ converges to $x$ in $E_{\mathrm{B}}$. Then, since $f \mid E_{B}$ is bounded, we must have $f(x)=0$. 
We previously noted that vector subspaces of even finite codimension in an ultrabornological space can fail to be ultrabornological. However, as we now proceed to show, hyperbornological spaces are much better behaved in this respect. Our main result in this direction is a strengthened, more general version of Corollary 1.3 in [13].

THEOREM 3.9. Let $E$ be a $\mathscr{L}$-bornological space, where $\mathscr{L}$ is a $b$-class with $\mathscr{L} \leq \mathscr{L}_{1}$. If $F$ is a vector subspace of countable codimension in $E$, then $F$ is hyperbornological.

Proof. By Theorem 1.2, $E$ is an inductive limit of normed vector spaces $\left\{E_{\lambda}: \lambda \in \Lambda\right\}$ belonging to $\mathscr{L}$; identifying each $E_{\lambda}$ with its image in $E$, put $F_{\lambda}=E_{\lambda} \cap F, \lambda \in \Lambda$. Since $\mathscr{L} \leq \mathscr{L}_{1}, E_{\lambda}$ is barrelled for every $\lambda \in \Lambda$, and therefore, because $F_{\lambda}$ has countable codimension in $E_{\lambda}$, the normed space $F_{\lambda}$ is barrelled (cf. [9]) for each $\lambda \in \Lambda$. But this means $F_{\lambda} \in \mathscr{L}_{t}, \lambda \in \Lambda$, while $F=\operatorname{ind}_{\lambda \rightarrow}\left\{F_{\lambda}: \lambda \in \Lambda\right\}$ by [13, Theorem 3]. Another application of Theorem 1.2 now serves to conclude the argument.

COROLLARY 3.10. If $E$ is hyperbornological, then so also is every vector subspace of countable codimension in $E$.

In passing, let us mention that, when taken in conjunction with Valdivia's work in [17], Theorem 3.9 provides a ready source of hyperbornological spaces which are not ultrabornological.

The final topic for discussion in the present section concerns stability under enlargements of the (continuous) dual. In the case of barrelled spaces, this question has previously been considered by several authors; we refer the reader to an article by W. J. Robertson and F. E. Yeomans [8] for additional details.

THEOREM 3.11. Let $E$ be a hyperbornological space. If $H$ is a finite dimensional vector subspace of $E^{*}$, then $E$ is also hyperbornological under the Mackey topology $\tau\left(E, E^{\prime}+H\right)$.

Proof. It will suffice to consider the one dimensional case, and so let us assume that $H$ is the linear span of a fixed functional $h \in E^{*} \backslash E^{\prime}$. Then, by Corollary 3.10, the hyperplane $F=\{x \in E: h(x)=0\}$ is hyperbornological in the topology induced by $\tau\left(E, E^{\prime}\right)$ which, in turn, must coincide with the topology $\tau\left(F, F^{\prime}\right)$. Let $G=E^{\prime}+H$. Then it is straightforward to check that the topology induced on $F$ by $\tau(E, G)$ is $\tau\left(F, E^{\prime}\right)$, which is to say $\tau\left(F, F^{\prime}\right)$. Thus, $(E, \tau(E, G))$ is the direct sum of (two) hyperbornological spaces and therefore itself hyperbornological by Proposition 3.1.

We next observe that, in some sense, the preceding theorem is the best possible.

THEOREM 3.12. If $E$ is hyperbornological and if $E^{*} \backslash E^{\prime} \neq \varnothing$, then there exists a countably infinite dimensional vector subspace $H$ of $E^{*}$ such that $\left(E, \tau\left(E, E^{\prime}+H\right)\right)$ is not hyperbornological.

Proof. Since not every $f \in E^{*}$ is bounded, $E$ contains a bounded set which is not a subset of any finite dimensional vector subspace of $E$. Consequently, there exists a countably infinite dimensional vector subspace $H$ of $E^{*}$ such that $\left(E, \tau\left(E, E^{\prime}+H\right)\right)$ is not barrelled [8, Theorem 2] whence not hyperbornological in view of Corollary 1.4. 
4. Closed graph theorems. Let $E$ and $F$ be Hausdorff locally convex spaces, and take $t: E \rightarrow F$ to be linear. Given a b-class $\mathscr{L}$, we will say that the graph of $t$ is $\mathscr{L}$-closed in $E \times F$ if, for every absolutely convex set $B \in \mathscr{L}(E), t \mid E_{\mathrm{B}}$ has a closed graph in $E_{B} \times F$. Of course, the graph of $t$ will always be $\mathscr{L}$-closed in $E \times F$ whenever it is closed. For convenience, we will refer to $E$ as having the $\mathscr{L}$-closed graph property (for Banach spaces) if, for any Banach space $F$, each linear mapping $t: E \rightarrow F$ having a $\mathscr{L}$-closed graph in $E \times F$ is necessarily continuous.

De Wilde [2], in a result analogous to the aforementioned theorem for barrelled spaces due to Mahowald [6], has shown that a Hausdorff locally convex space having the $\mathscr{L}_{\mathrm{B}}$-closed graph property is ultrabornological. As we next note, there is a parallel statement for arbitrary b-classes.

Propostrion 4.1. For any $b$-class $\mathscr{L}$, if $E$ is a Hausdorff locally convex space which has the $\mathscr{L}$-closed graph property, then $E$ is $\mathscr{L}$-bornological.

Proof. (DeWilde's argument is equally applicable to this more general situation, but we shall include a brief sketch for the sake of completeness.) Let $U$ be an absolutely convex subset of $E$ which absorbs every $A \in \mathscr{L}(E)$, take $p$ to be the gauge of $U$, put $G=E / p^{-1}(0)$ equipped with the norm induced by $p$, and denote the completion of $G$ by $F$. Then the projection $t$ of $E$ onto $G$ has a $\mathscr{L}$-closed graph in $E \times F$ whereby $t$ is continuous, and $U$ is therefore a neighborhood in $E$.

In the converse direction, we have the following generalization of the closed graph theorem for ultrabornological spaces originally established in [2].

THeOREM 4.2. If $\mathscr{L}$ is a b-class with $\mathscr{L} \leq \mathscr{L}_{1}$ and if $E$ is any $\mathscr{L}$-bornological space, then $E$ has the $\mathscr{L}$-closed graph property.

Proof. Take any linear mapping $t$ from $E$ into a Banach space $F$ such that the graph of $t$ is $\mathscr{L}$-closed in $E \times F$. In view of Theorem 1.2 , it will suffice to show that $t_{\mathrm{B}}=t \mid E_{\mathrm{B}}$ is continuous, where $B$ is an absolutely convex bounded set in $E$ for which $E_{B}$ is topologically isomorphic to a member of $\mathscr{L}$. However, since $E_{B}$ is thus barrelled and $t_{B}$ has a closed graph in $E_{B} \times F$, this must indeed be the case (cf. [7, p. 116]).

One immediate consequence of 4.1 and 4.2 is a further characterization of hyperbornological spaces.

THEOREM 4.3. A Hausdorff locally convex space $E$ is hyperbornological if, and only if, $E$ has the $\mathscr{L}_{1}$-closed graph property.

Interestingly enough, and another argument in favor of hyperbornological spaces, Theorem 4.2 is the best possible in that the converse assertion is also valid.

TheOREm 4.4. Given a $b$-class $\mathscr{L}$, if every $\mathscr{L}$-bornological space has the $\mathscr{L}$-closed graph property, then $\mathscr{L} \leq \mathscr{L}_{\text {. }}$.

Proof. Fixing $E \in \mathscr{L}$, let $F$ be a Banach space, and assume that $t: E \rightarrow F$ is a linear mapping having a closed graph in $E \times F$. Since the graph of $t$ is then $\mathscr{L}$-closed, and since $E$ 
is $\mathscr{L}$-bornological, $t$ is continuous whereby $E$ is barrelled in view of Mahowald's characterization [6].

5. Observations and examples. The b-class $\mathscr{L}_{r}$ was introduced in Section 1 , but further consideration was then deferred. We now return to pick up that thread.

For any two subsets $\mathscr{E}$ and $\mathscr{F}$ of $\mathcal{N}$, let us agree to also write $\mathscr{E} \leq \mathscr{F}$ whenever each $E \in \mathscr{E}$ is topologically isomorphic to some $F \in \mathscr{F}$. Then, given $\mathscr{E} \subseteq \mathcal{N}$, there is a (unique) b-class $\mathscr{L}_{\mathscr{\varnothing}}$ such that (a) $\mathscr{E} \leq \mathscr{L}_{\mathscr{\varnothing}}$ and (b) $\mathscr{L}_{\varnothing} \leq \mathscr{L}$ for every b-class $\mathscr{L}$ with $\mathscr{C} \leq \mathscr{L}$; we will refer to $\mathscr{L}_{\mathscr{G}}$ as the $b$-class generated by $\mathscr{E}$. In particular, we will let $\mathscr{L}_{\mathrm{h}}$ denote the b-class generated by the singleton subset $\mathscr{E}$ of $\mathcal{N}$ consisting of the separable Hilbert space $l^{2}$.

In [16], Valdivia established that every ultrabornological space is an inductive limit of members of $\mathscr{L}_{h}$, and the next result is an easy consequence of this fact.

Proposition 5.1. The following are equivalent for a Hausdorff locally convex space $E$ :

(i) $E$ is $\mathscr{L}_{h}$-bornological;

(ii) $E$ is $\mathscr{L}_{\mathrm{r}}$-bornological;

(iii) $E$ is ultrabornological.

Thus, while $\mathscr{L}_{r}$ and $\mathscr{L}_{B}$ are distinct b-classes, the $\mathscr{L}_{r}$-bornological spaces coincide with the $\mathscr{L}_{B}$-bornological spaces. Moreover, among b-classes which determine the ultrabornological spaces, the b-class $\mathscr{L}_{\mathrm{h}}$ is in some sense minimal. These observations suggest the possibility of minimal or maximal b-classes being associated with a given b-class $\mathscr{L}$, and we now proceed to address this question.

If $\mathscr{L}$ is a b-class, then there is a natural candidate for an associated maximal b-class; put

$$
\mathcal{M}(\mathscr{L})=\{E \in \mathcal{N}: E \text { is } \mathscr{L} \text {-bornological }\} .
$$

In view of Proposition 3.1, $M(\mathscr{L})$ is clearly the right choice, but let us make this more explicit.

Proposirion 5.2. If $\mathscr{L}$ is a $b$-class, then $M(\mathscr{L})$ is a $b$-class, and indeed the only one, satisfying the following properties:

(a) every $\mathcal{M}(\mathscr{L})$-bornological space is $\mathscr{L}$-bornological;

(b) if $\mathscr{L}^{\#}$ is a b-class such that every $\mathscr{L}^{\#}$-bornological space is $\mathscr{L}$-bornological, then $\mathscr{L}^{\#} \leq \mathcal{M}(\mathscr{L})$.

Besides the case for $\mathcal{N}$ itself, it is also obvious that $\mathscr{L}_{f}=\mathcal{M}\left(\mathscr{L}_{f}\right)$ and $\mathscr{L}_{t}=\mathcal{M}\left(\mathscr{L}_{t}\right)$. However, because there do exist ultrabornological normed spaces which are not complete (cf. [3]), $\mathscr{L}_{B}$ is not maximal. In fact, as we next observe, the example of an incomplete barrelled normed vector space given by Köthe [5, p. 369] is actually ultrabornological.

EXAMPLE 5.3. Recalling that a sequence $\phi: \mathbb{N} \rightarrow \mathbb{N}$ has density zero if $\lim _{n \rightarrow \infty} \frac{n}{\phi(n)}=0$, let $E$ be the (dense) vector subspace of $l^{1}$ consisting of all $g \in l^{1}$ which vanish off a sequence of density zero, and equip $E$ with the topology induced by the $l^{1}$-norm. Now, 
choose $f \in E^{*}$ such that $f$ is $\mathscr{L}_{\mathrm{B}}$-locally bounded, and consider the sequence $\left\{e_{k}\right\} \subseteq E$, where $e_{k}(n)=\delta_{k n}, k, n=1,2, \ldots$ If the sequence $\left\{f\left(e_{k}\right)\right\}$ were not bounded, then there would exist a sequence $\phi: \mathbb{N} \rightarrow \mathbb{N}$ of density zero such that $\left|f\left(e_{\phi(k)}\right)\right| \geq k, k=1,2, \ldots$ Then, putting $E_{\phi}=\left\{g \in l^{1}: g(\mathbb{N} \backslash \phi(\mathbb{N}))=0\right\}$, we have $E_{\phi} \subseteq E$ and $E_{\phi}$ is a Banach space under the induced topology whereby $f \mid E_{\phi}$ is continuous by Corollary 2.3. But this is clearly contradictory, and so there exists $\beta>0$ so that $\left|f\left(e_{k}\right)\right| \leq \beta, k \in \mathbb{N}$. Fixing $g \in E$, let $\phi: \mathbb{N} \rightarrow \mathbb{N}$ be a sequence of density zero such that $g(\mathbb{N} \backslash \phi(\mathbb{N}))=0$. As above, $f \mid E_{\phi}$ is continuous whence $|f(g)| \leq \beta\|g\|_{1}$, i.e. $f \in E^{\prime}$. Since $E$ is barrelled, $E$ has its Mackey topology $\tau\left(E, E^{\prime}\right)$, and thus Corollary 2.3 applies to show that $E$ is ultrabornological.

Minimality is less straightforward. Of course, besides being maximal, $\mathscr{L}_{f}$ is minimal by definition, but this is certainly a special case. As to $\mathscr{L}_{B}$, while $\mathscr{L}_{h}$ can be regarded as an associated minimal b-class, uniqueness is ruled out by Theorem 4 in [16], and we do not know whether $\mathscr{L}_{t}$ even has an associated minimal b-class.

The following example should help clarify the position occupied by the generalized barrelledness properties which we introduced in the first section.

Example 5.4. Let $E$ denote the vector subspace of $l^{\infty}$ consisting of all absolutely summable sequences equipped with the relative supremum norm topology, and consider the b-class $\mathscr{L}_{\&}$ generated by $\mathscr{E}=\{E\}$. Then $E$ is $\mathscr{L}_{\mathscr{C}}$-quasibarrelled, but $E$ is not barrelled since $l^{1}$ can not have a strictly coarser Hausdorff barrelled topology (cf. [7, p. 117]). If we next take $F$ to be the vector subspace of $E$ consisting of those $g \in E$ which vanish off a finite subset of $\mathbb{N}$ and give $F$ the topology induced by $E$, then $F$ is quasibarrelled. If $G \in \mathscr{L}_{\mathscr{B}}$, however, then there is a corresponding closed vector subspace $M$ of $E$ such that $G$ is topologically isomorphic to $E / M$ whereby each $G \in \mathscr{L}_{\&}$ is a Banach space under the quotient norm induced by $l^{1}$. Therefore, because $\mathscr{L}_{\&}$ contains no elements having countably infinite dimension, $E_{\mathrm{B}}$ is finite dimensional whenever $B$ is an absolutely convex member of $\mathscr{L}_{\mathscr{B}}(F)$. Since any barrel in $F$ will thus absorb every $A \in \mathscr{L}_{\mathscr{G}}(F)$, we have that $F$ is not $\mathscr{L}_{8}$-quasibarrelled.

While not every bornological and barrelled Hausdorff locally convex space is hyperbornological, our characterization in terms of the $\mathscr{L}_{t}$-closed graph property (Theorem 4.3) suggests asking whether bornological spaces with what one might term the "sequentially closed graph property" are necessarily hyperbornological. We shall show that even this is not the case.

EXAmPLE 5.5. Take $E$ to be the example of an incomplete (LB)-space given by Köthe $[5$, p. 434]. Then, since $E$ actually fails to be sequentially complete, there exists $y \in \hat{E} \backslash E$, where $\hat{E}$ denotes the completion of $E$, and a sequence $\left\{x_{n}\right\}$ in $E$ such that $\left\{x_{n}\right\}$ converges to $y$ in $\hat{E}$; let $F$ be the linear span of $E \cup\{y\}$ with the topology inherited from $\hat{E}$. Valdivia [15] has shown that $F$ is a bornological (and barrelled) space which is not hyperbornological, and so we only need verify that $F$ satisfies the appropriate closed graph property. To this end, let $G$ be a Banach space, and consider a linear mapping $t: F \rightarrow G$ which has a sequentially closed graph in $F \times G$. Since $E$ is hyperbornological, $t \mid E$ is continuous by Theorem 4.3. Thus, taking $f: F \rightarrow G$ to be the continuous linear extension of $t \mid E$, we have that the sequence $\left\{t\left(x_{n}\right)\right\}$ converges to $f(y)$ wherefore $t(y)=f(y)$, i.e. $t$ is continuous. 


\section{REFERENCES}

1. H. H. Corson, Normality in subsets of product spaces, Amer. J. Math. 81 (1959), 785-796.

2. M. De Wilde, Ultrabornological spaces and the closed graph theorem, Bull. Soc. Roy. Sci. Liège 40 (1971), 116-118.

3. S. Dierolf and P. Lurje, Deux exemples concernant des espaces (ultra) bornologiques, $C . R$. Acad. Sci. 282 (1976), 1347-1350.

4. H. Hogbe-Nlend, Théorie des bornologies et applications, Lecture Notes in Mathematics 213 (Springer, 1971).

5. G. Köthe, Topological vector spaces I (Springer, 1969).

6. M. Mahowald, Barrelled spaces and the closed graph theorem, J. London Math. Soc. 36 (1961), 108-110.

7. A. P. Robertson and W. J. Robertson, Topological vector spaces (Cambridge, 1964).

8. W. J. Robertson and F. E. Yeomans, On the stability of barrelled topologies, I, Bull. Austral. Math. Soc. 20 (1979), 385-395.

9. S. Saxon and M. Levin, Every countable-codimensional subspace of a barrelled space is barrelled, Proc. Amer. Math. Soc. 29 (1971), 91-96.

10. L. Schwartz, Sur le théorème du graphe fermé, C. R. Acad. Sci. 263 (1966), 602-605.

11. M. Valdivia, Absolutely convex sets in barrelled spaces, Ann. Inst. Fourier (Grenoble) 21 (1971), 3-13.

12. M. Valdivia, A class of bornological barrelled spaces which are not ultrabornological, Math. Ann. 194 (1971), 43-51.

13. M. Valdivia, On final topologies, J. Reine Angew. Math. 251 (1971), 193-199. $27-30$.

14. M. Valdivia, On nonbornological barrelled spaces, Ann. Inst. Fourier (Grenoble) 22 (1972),

15. M. Valdivia, Some properties of the bornological spaces, Publ. Dép. Math. (Lyon) 10 (1973), 51-56.

16. M. Valdivia, A class of precompact sets in Banach spaces, J. Reine Angew. Math. 276 (1975), 130-136.

17. $M$. Valdivia, Sur certains hyperplans qui ne sont pas ultra-bornologiques dans les espaces ultra-bornologiques, C. R. Acad. Sci. Sér. A 284 (1977), 935-937.

Department of Mathematics

JADAVPUR UNIVERSTTY

CALCUTTA-700009

INDIA
Department of Mathematics UNIVERSTTY OF ARKANSAS

FAYETTEVILLE, AR 72701

USA 